\title{
Demonstration of sensory innervation of rat tongue with anterogradely transported horseradish peroxidase
}

\author{
ROBERT M. BRADLEY $Y^{1,2}$, CHARLOTTE M. MISTRETTA ${ }^{1,3}$ and TAKATOSHI NAGAI ${ }^{1, *}$ \\ 'Department of Oral Biology, University of Michigan, School of Dentistry, Ann Arbor, MI 48109; 2The Department of Physiology, \\ School of Medicine, University of Michigan, Ann Arbor, MI 48109; ${ }^{3}$ The Center for Nursing Research, School of Nursing, and The \\ Center for Human Growth and Development, University of Michigan, Ann Arbor, MI 48109 (U.S.A.)
}

(Accepted November 5th, 1985)

Key words: horseradish peroxidase - anterograde transport - glossopharyngeal nerve - taste bud

\begin{abstract}
Wheat germ agglutinin-horseradish peroxidase injected into the petrosal ganglion in rats was transported to the posterior tongue in the glossopharyngeal nerve. Labelled fibers innervated the ipsilateral foliate papilla only, but both ipsi- and contralateral sides of the single circumvallate papilla. Somatosensory fibers in the posterior tongue also were labelled. Label crossed transcellularly from the afferent fibers into taste bud cells. Use of this method provides more detailed knowledge of sensory innervation of the tongue than previously available.
\end{abstract}

Previous studies of lingual gustatory innervation have used electron microscopy or light microscopy with various silver stains, sometimes in combination with selective degeneration of peripheral nerves ${ }^{3,9}$. Although these have provided general information on tongue innervation, the methods are non-selective. Without associated nerve cutting, they cannot demonstrate the extent of bilateral innervation or distinguish between afferent and efferent nerve fibers; nor can they be used for tracing sensory nerve endings from a defined or limited group of ganglion cells. Recently published reports indicate the usefulness of anterograde axoplasmic transport of horseradish peroxidase (HRP) to selectively demonstrate a particular group of terminal sensory fibers ${ }^{5-7,13}$. In these studies, HRP conjugated with wheat germ agglutinin (WGA-HRP) was transported anterogradely from sensory ganglia and used to trace terminal branches of sensory nerves. We have applied this technique to trace the peripheral connections of the gustatory innervation of the tongue. Injections were made into the petrosal ganglion, which contains the cell bodies of glossopharyngeal nerve fibers that in- nervate the posterior tongue and taste buds in circumvallate and foliate papillae.

Adult male, Sprague-Dawley rats, weighing 125-250 g, were used for these experiments. The final series of experiments involved 8 rats. Numerous other animals were used in initial studies to optimize fixation of the tongue and the injection technique. It was found that adequate fixation of the tongue, with subsequent limited precipitation of the chromagen, could only be obtained when the highest purity paraformaldehyde was used. This was essential for resultant low background and visualization of nerve fibers.

Rats were anesthetized via an i.p. injection of sodium pentobarbital ( $50 \mathrm{mg} / \mathrm{kg} \mathrm{b}$. wt.) and given supplemental doses as necessary. The petrosal ganglion was exposed and $3 \mu \mathrm{l}$ of $4 \% \mathrm{WGA}-\mathrm{HRP}$ in saline was injected slowly with pressure from a micropipette $(\sim 40 \mu \mathrm{m}$ diameter $)$. The incisions were closed and the animals allowed to recover. After a postoperative survival time of $6-24 \mathrm{~h}$ the rats were anesthetized and perfused transcardially. The blood was flushed from the vascular system with $0.1 \mathrm{M}$ phos-

\footnotetext{
* Present address: Department of Physiology, Teikyo University, School of Medicine, Kaga 2-11-1, Itabashi-ku, Tokyo, Japan. Correspondence: R. M. Bradley, Department of Oral Biology, School of Dentistry, University of Michigan, Ann Arbor, MI 48109, U.S.A.
} 
phate buffer containing $0.9 \%$ sodium chloride, $3 \%$ procaine $\mathrm{HCl}$ and $0.003 \%$ heparin, followed by a solution of $4 \%$ glutaraldehyde and $1 \%$ paraformaldehyde in $0.1 \mathrm{M}$ phosphate buffer ( $\mathrm{pH} 7.3$ ), and finally by cold $10 \%$ sucrose in $0.1 \mathrm{M}$ phosphate buffer. The tongue was removed and stored for $24 \mathrm{~h}$ in cold $10 \%$ sucrose in $0.1 \mathrm{M}$ phosphate buffer. Serial, $40 \mu \mathrm{m}$ or $60 \mu \mathrm{m}$ frozen sections of the posterior tongue were cut in the coronal plane on a sliding microtome. The tissue was treated with tetramethyl benzidine as a chromagen for the demonstration of HRP reaction product $^{8}$. Sections were mounted out of cold $0.1 \mathrm{M}$ acetate buffer ( $\mathrm{pH}$ 3.3) onto chrome-alum-subbed slides. All sections were examined using both lightfield and darkfield microscopy. Selected sections were photographed.

Examination of the posterior tongue revealed intense labelling of the glossopharyngeal nerve branches that innervate the circumvallate and foliate papillae. Labelled fibers innervated the ipsilateral foliate papilla only, but both ipsi- and contralateral sides of the single circumvallate papilla (Fig. 1a).

The fibers of the glossopharyngeal nerve innervating the circumvallate papilla entered the base of the papilla laterally. These fibers divided into a branch supplying the taste buds of the ipsilateral lateral wall
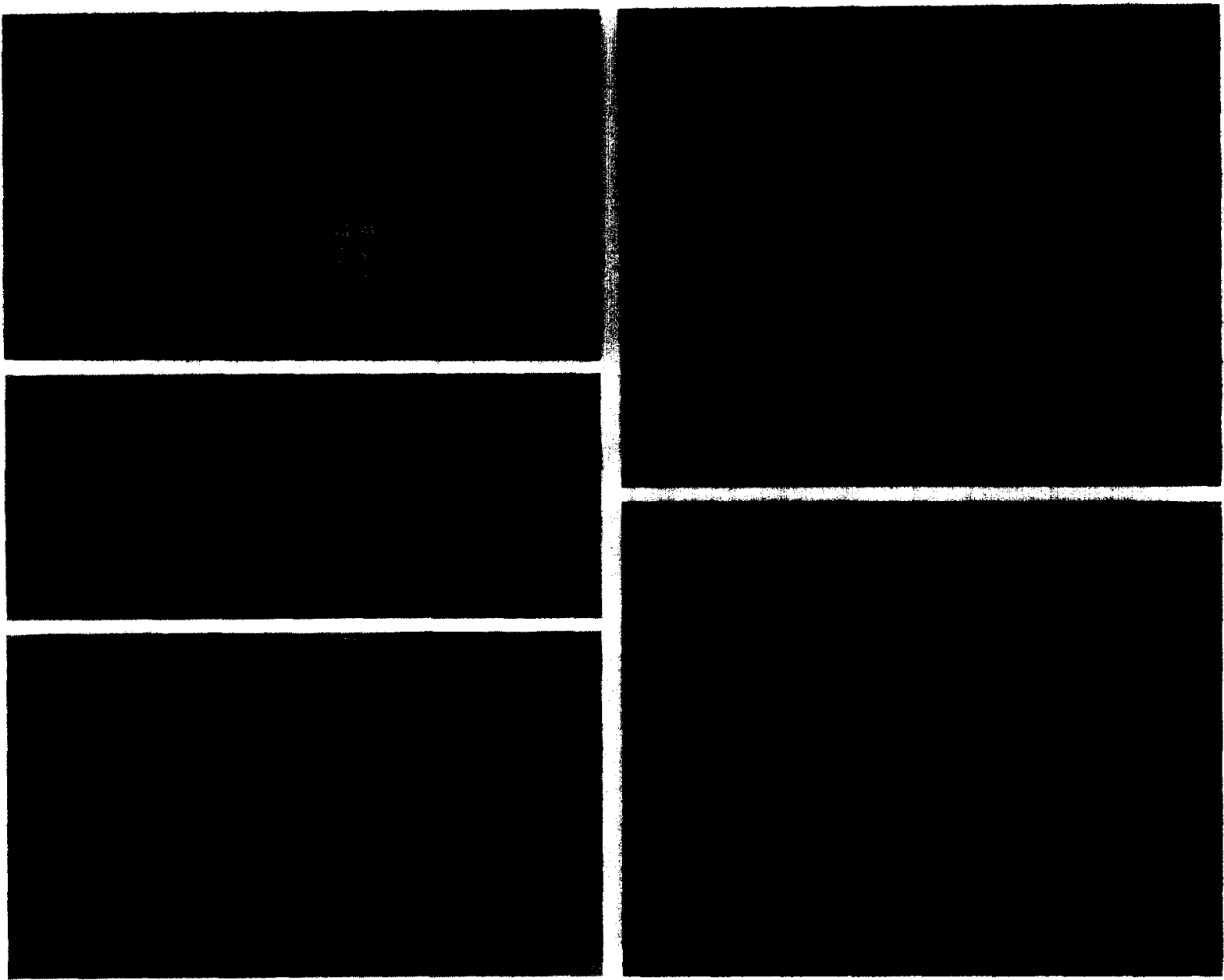

Fig. 1 a: coronal section through the centrally located, single circumvallate papilla showing both ipsi- and contralateral labelling (dark field, $\mathrm{bar}=100 \mu \mathrm{m})$; $\mathrm{b}$ : light field photomicrograph of the dorsal surface of the circumvallate papilla with two labelled somatosensory endings (bar $=100 \mu \mathrm{m}$ ); : light field photomicrograph showing single taste bud cells (arrows) filled with reaction product (bar $=50$ $\mu \mathrm{m})$; $\mathrm{d}$ : coronal section through the circumvallate papilla showing a lateral branch of the glossopharyngeal nerve innervating the ipsilateral wall of the papilla and a branch ascending the central core of the papilla (dark field, bar $=100 \mu \mathrm{m}$ ); e: coronal section through the circumvallate papilla showing fascicles from the central branch looping downwards below the trough epithelium (arrow) to reach and innervate taste buds of the contralateral papilla wall (dark field, bar $=100 \mu \mathrm{m}$ ). 
of the papilla and a larger branch which passed up the center of the papilla core (Fig. 1d). Fascicles from this central branch looped downwards below the trough epithelium to reach and innervate the taste buds of the contralateral papilla wall (Fig. 1e). Presumably these looping fibers were following unlabelled contralateral axons a short distance in a retrograde direction to reach the contralateral taste buds.

Somatosensory fibers in the posterior tongue also were labelled. Fibers from the central and lateral branches in the circumvallate papilla terminated as Merkel cell endings in the superficial epithelium of the papilla and surrounding epithelium. In the papilla these mechanoreceptive endings were found on both ipsilateral and contralateral sides (Fig. 1b) and were probably bilaterally innervated, also.

The anterograde transport of HRP was rapid and reached the tongue within $6 \mathrm{~h}$ of the injection. With such a short survival time the label was less dense than with $18-24 \mathrm{~h}$ survivals and it was possible to follow individual fibers.

A very interesting result of these studies was the demonstration of label within the taste bud cells. Fig. 1c clearly shows cells of the taste bud that are outlined by reaction product. This was not the result of endogenous peroxidase activity because the contralateral foliate papilla was devoid of any label. The label was transported to the taste bud cells via the nerve supply since taste buds are avascular structures. Any peroxidase entering blood vessels contained in the glossopharyngeal nerve would have been either removed from the circulation during the time between injection and sacrifice or at the time of the perfusion.

The reaction product was within the cell cytoplasm and the position of the cell nucleus was revealed as a clear area (arrows in Fig. 1c). Since taste bud cells are specialized epithelial cells which are in synaptic contact with gustatory nerve terminals ${ }^{10}$, the label crossed from the sensory afferents into the taste bud cells. HRP is a large molecule and usually does not pass transcellularly ${ }^{8}$. It is apparent from the present experiments that HRP can pass transcellularly from the gustatory afferents to the taste bud cells. The taste afferents are therefore unusually permeable to HRP and the taste bud cells are able to take up the released tracer molecules. This uptake is specific to the taste bud (and Merkel cells) since surrounding epi- thelial cells do not take up the label. It is interesting that in other unrelated experiments, in which WGAHRP was injected directly into the tongue in the area of the circumvallate papilla, the taste buds also were observed to have incorporated the label (unpublished observations).

It is not possible with light microscopy to determine if the label is in a particular cell type in the taste bud. This would require examination of the labelled taste buds with electron microscopy.

The extensive bilateral innervation of the circumvallate papilla demonstrated with WGA-HRP labelling is not surprising since secticn of one glossopharyngeal nerve only results in a 5-12\% loss of taste buds in the circumvallate papilla ${ }^{4,12,14}$. The route taken by the fibers of one glossopharyngeal nerve to reach the contralateral taste buds is interesting, since it is somewhat indirect and travels with the contralateral nerve.

The use of this technique illustrates the complexity of the innervation of the circumvallate papilla with extensive bilateral innervation of taste and somatosensory receptors. Moreover, the dense meshwork of fibers beneath the gustatory epithelium suggests that fibers branch to supply several taste buds and taste cells within a taste bud. Electron microscope studies of the rat fungiform papilla also have revealed considerable complexity ${ }^{1}$. Furthermore, recent studies on the localization of substance $\mathrm{P}$ in the rat circumvallate papilla have shown that perigemmal fibers contain substance P-like immunoreactive material and that these immunoreactive perigemmal fibers sometimes penetrate into the taste bud ${ }^{11.15}$. The results of these neuroanatomical techniques suggest that the circumvallate papilla is a complex sensory organ with its own set of sensory receptors and neurotransmitters and is possibly involved in the first stage of sensory integration at the level of the tongue.

The method of anterograde HRP transport has potential for tracing peripheral innervation of a selected group of ganglion cells. Previous studies have used transported tritiated amino acids to demonstrate peripheral nerve axons ${ }^{2}$. The use of HRPWGA tracing has a number of advantages including technical simplicity, rapid results and visualization of the label in the tissue (not in a photographic emulsion above the tissue), resulting in greater resolution. Our ultimate goal with this technique is to examine the 
terminal fields of single taste afferents. This will require a more limited injection site to restrict the number of ganglion cells labelled or perhaps intracellular injection of WGA-HRP. Both of these techniques are possible and will extend the usefulness of employing WGA-HRP to study peripheral innervation.

1 Beidler, L.M. Innervation of rat fungiform papilla. In C. Pfaffmann (Ed.), Olfaction and Taste, Vol. III, Rockefeller University Press, New York, 1969, pp. 352-369.

2 Byers, M.R., Development of sensory innervation in dentin, J. Comp. Neurol., 191 (1980) 413-427.

3 Farbman, A. I. and Hellekant, G., Quantitative analyses of the fiber population in rat chorda tympani nerves and fungiform papillae, Am. J. Anat., 153 (1978) 509-522.

4 Guth, L., Histological changes following partial denervation of the circumvallate papilla of the rat, Exp. Neurol., 8 (1963) 336-349.

5 Kiang, N.Y.S., Rho, J.M., Northrop, C.C., Liberman, M.C. and Ryugo, D.K., Hair-cell innervation by spiral ganglion cells in adult cats, Science, 217 (1982) 175-177.

6 Liberman, M.C., Single-neuron labeling in the cat auditory nerve, Science, 216 (1982) 1239-1240.

7 Marfurt, C.F. and Turner, D.F., Sensory nerve endings in the rat orofacial region labeled by the anterograde and transganglionic transport of horseradish peroxidase: a new method for tracing peripheral nerve fibers, Brain Research, 261 (1983) 1-12.

8 Mesulam, M.-M., Tracing Neural Connections with Horse-
This work was supported by N.S.F. grant BNS 80-15737 to C.M.M. and R.M.B. We thank Dr. Dennis $F$. Turner for help and advice on the technical aspects of this work.

radish Peroxidase, Wiley, New York, 1982, 247 pp.

9 Miller, I.J., Gomez, M.M. and Lubarsky, E.H., Distribution of the facial nerve to taste receptors in the rat, Chem. Senses Flav., 3 (1978) 397-411.

10 Murray, R.G., The ultrastructure of taste buds. In I. Friedmann (Ed.), The Ultrastructure of Sensory Organs, Elsevier/North-Holland Amsterdam, 1973, pp. 1-82.

11 Nagy, J.I., Goedert, M., Hunt, S.P. and Bond, A., The nature of the substance P-containing nerve fibers in taste papillae of the rat tongue, Neuroscience, 7 (1982) 3137-3151.

12 Oakley, B., On the specification of taste neurons in the rat tongue, Brain Research, 75 (1974) 85-96.

13 Robertson, B. and Aldskogius, H., The use of anterogradely transported wheat germ agglutinin-horseradish peroxidase conjugate to visualize cutaneous sensory nerve endings, Brain Research, 240 (1982) 327-330.

14 Whiteside, B., Nerve overlap in the gustatory apparatus of the rat, J. Comp. Neurol., 44 (1927) 363-377.

15 Yamasaki, H., Kubota, Y., Takagi, H. and Tohyama, M., Immunoelectronmicroscopic study on the fine structure of substance P-containing fibers in the taste buds of the rat, J. Comp. Neurol., 227 (1984) 380-392. 